

\title{
Statistics of injected power on a bouncing ball subjected to a randomly vibrating piston
}

\author{
Alfredo García-Cid, Pablo Gutiérrez, Claudio Falcón, Sébastien Aumaître, \\ Eric Falcon
}

\section{- To cite this version:}

Alfredo García-Cid, Pablo Gutiérrez, Claudio Falcón, Sébastien Aumaître, Eric Falcon. Statistics of injected power on a bouncing ball subjected to a randomly vibrating piston. Physical Review E , 2015, 92, pp.032915. 10.1103/PhysRevE.92.032915 . cea-01367017

\section{HAL Id: cea-01367017 https://hal-cea.archives-ouvertes.fr/cea-01367017}

Submitted on 15 Sep 2016

HAL is a multi-disciplinary open access archive for the deposit and dissemination of scientific research documents, whether they are published or not. The documents may come from teaching and research institutions in France or abroad, or from public or private research centers.
L'archive ouverte pluridisciplinaire HAL, est destinée au dépôt et à la diffusion de documents scientifiques de niveau recherche, publiés ou non, émanant des établissements d'enseignement et de recherche français ou étrangers, des laboratoires publics ou privés. 


\title{
Statistics of injected power on a bouncing ball subjected to a randomly vibrating piston
}

\author{
Alfredo García-Cid, Pablo Gutiérrez, and Claudio Falcón* \\ Departamento de Física, Facultad de Ciencias Físicas y Matemáticas, Universidad de Chile, Casilla 487-3, Santiago, Chile \\ Sébastien Aumaître \\ Service de Physique de l'Etat Condensé, DSM, CEA-Saclay, UMR 3680 CNRS, 91191 Gif-sur-Yvette, France \\ and Laboratoire de Physique, UMR-CNRS 5672, Ecole Normale Supérieure de Lyon, 46 allée d'Italie, Lyon, France
}

Eric Falcon

Univ Paris Diderot, Sorbonne Paris Cité, MSC, UMR 7057 CNRS, F-75 013 Paris, France

(Received 30 March 2015; published 29 September 2015)

\begin{abstract}
We present an experimental study on the statistical properties of the injected power needed to maintain an inelastic ball bouncing constantly on a randomly accelerating piston in the presence of gravity. We compute the injected power at each collision of the ball with the moving piston by measuring the velocity of the piston and the force exerted on the piston by the ball. The probability density function of the injected power has its most probable value close to zero and displays two asymmetric exponential tails, depending on the restitution coefficient, the piston acceleration, and its frequency content. This distribution can be deduced from a simple model assuming quasi-Gaussian statistics for the force and velocity of the piston.
\end{abstract}

DOI: 10.1103/PhysRevE.92.032915

PACS number(s): 05.45.-a, 47.20.Ky

\section{INTRODUCTION}

Dissipative systems display striking dynamical behaviors when they are driven by an external forcing. A nonequilibrium steady state (NESS) can be achieved by balancing the effect of the external forcing and the inherent dissipative mechanisms of the system under study. In physics, the number of examples is vast. For instance, in fluid mechanics, a typical example of NESS is the so-called fully developed hydrodynamic turbulence $[1,2]$, where the energy pumped at a large scales is transported towards small scales where it is dissipated by viscosity. In granular materials, a granular gas is a NESS achieved by injecting energy through the boundaries of a container into a dilute sample of particles which will later dissipate it via inelastic collisions, converting kinetic energy into plastic deformations [3-6]. Similar examples of nonequilibrium steady states can be found in electronic circuits [7-9], turbulent thermal convection [10], two-dimensional turbulence [11], wave turbulence in elastic plates [12] and surface waves [13], molecular machines [14], and so forth. In these steady states, the injected power or other global quantities (i.e., quantities averaged over space and/or time such as energy, momenta, and number of particles) will fluctuate strongly and depend on both the injection mechanism and the impedance of the system to the driving. Thus, in a NESS these global quantities cannot be regarded as constant as they depend on the interaction between the system and the injection mechanism, even in the case of time-periodic (sinusoidal) forcing [15]. When the driving can be described statistically by a Gaussian random process with zero mean, their fluctuations can be several times larger than their mean values [5,6,11-13]. It prevents the use of a large set of theoretical tools which are of fundamental use in the thermodynamical limit [16,17] which allow the prediction and control of systems with a large degree of components.

\footnotetext{
*cfalcon@ing.uchile.cl
}

A simple example of how an out-of-equilibrium balance between injection and dissipation generates a NESS is an inelastic bouncing ball driven vertically by a randomly moving piston in the presence of gravity. In this simple system energy is pumped into the bouncing ball by the moving piston, which is dissipated mainly by plastic deformations of the bead and viscous drag with the air surrounding the ball. The dynamics of inelastic bouncing balls excited by vertical vibrations has been researched extensively theoretically, experimentally, and numerically as examples of permanent chaotic behavior when the piston moves periodically [18-21]. On the other hand, only a handful of theoretical studies have reported on the properties and features of the NESS dynamics of the inelastic bouncing ball system when the motion of the piston is quasiperiodic [22] or when the bead is subjected to a random acceleration [23]. It must be noticed that, experimentally, the injection of energy (or other quantities) into a dissipative system is usually accompanied by fluctuations, affecting the statistical properties of the NESS under study. Thus, an experimental study on the properties and features of the NESS achieved by a randomly excited inelastic bouncing ball is lacking.

In the present paper, we focus our work on the statistics of the injected power furnished by the randomly moving piston which is necessary to maintain an inelastic ball bouncing constantly. In Sec. II we present the experimental setup and measuring techniques used to quantify the injected power $I_{n}$ on each impact of the bouncing ball. Section III shows the experimentally measured probability distribution functions (PDF) for the force $F_{n}$ and velocity $v_{n}$ of the moving piston as the bead makes the $n$th impact onto its surface, and with this data we compute the PDF of $I_{n}$. This distribution has its most probable value close to zero and displays two asymmetric exponential tails, depending on the restitution coefficient, the piston acceleration, and its frequency content. In Sec. IV we show that by using a simple modified bivariate distribution for $F_{n}$ and $v_{n}$ we can be compute theoretically the distribution of $I_{n}$. Finally, in Sec. V we discuss the possible applications of the model and how it can be generalized to granular gases. 


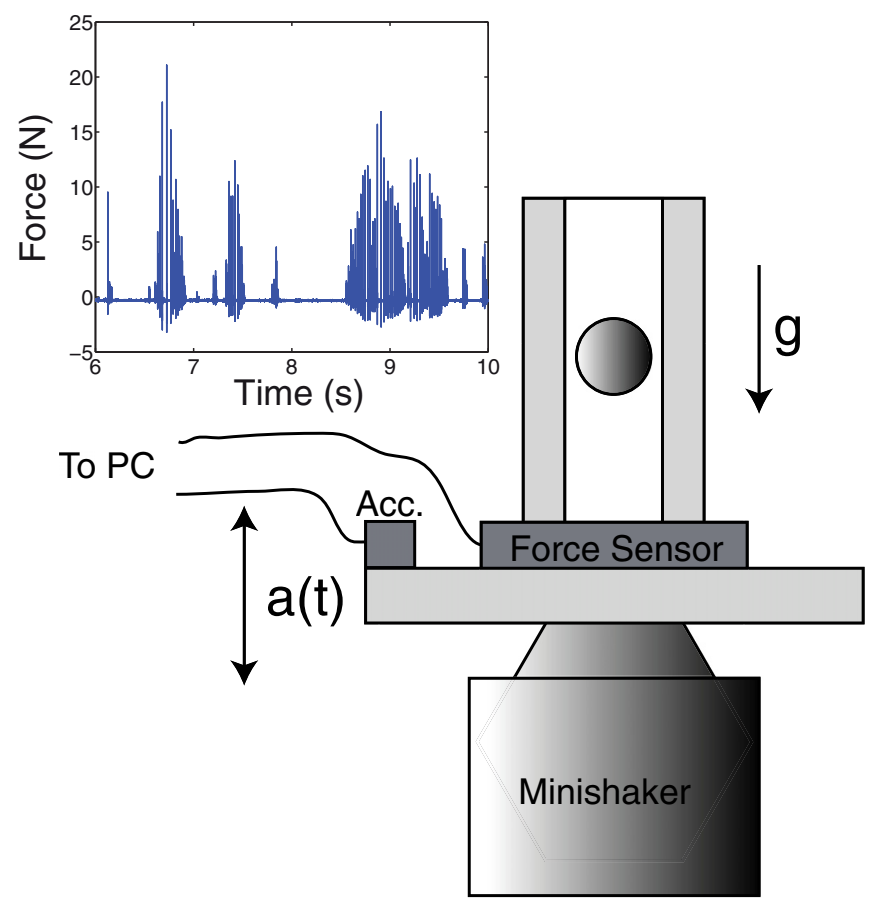

FIG. 1. (Color online) Experimental setup. Inset: Typical temporal trace of the force signal for a steel ball (ball mass $m=8 \mathrm{~g}$, restitution coefficient $r=0.923$ ) at a given control acceleration $\Gamma=1.09$.

\section{EXPERIMENTAL SETUP AND MEASUREMENT TECHNIQUES}

The experimental setup is shown in Fig. 1. An $8 \mathrm{~mm}$ bead (made out of steel, brass, copper, polyurethane, or nylon, with $\pm 1 \mu \mathrm{m}$ tolerance in diameter, $\pm 1 \mu \mathrm{m}$ in sphericity with masses presented in Table I) is placed inside a vertical plexiglass enclosing tube (outer diameter $15 \mathrm{~mm}$, inner diameter $9 \mathrm{~mm}$ ), which is polished on its inside part to reduce sliding friction with the bead. The tube is screwed to a plexiglass piston driven randomly in time by a minishaker (B\&K 4810). An accelerometer (B\&K 4803) screwed to the oscillating piston measures the acceleration $a(t)$ of the whole setup via a charge amplifier (B\&K 2635). The random forcing of the plexiglass piston, generated by a desktop computer using LabView via an acquisition card (NI-6022) through a power amplifier (B\&K 2706), is a zero-mean colored Gaussian noise with a controlled frequency bandwidth $\left[f_{l}, f_{u}\right]$ and rms amplitude normalized with respect to gravity $\Gamma=\sqrt{\left\langle a(t)^{2}\right\rangle} / g$. In our experiments $f_{u}$

TABLE I. Masses and COR for different materials using spheres of $8 \mathrm{~mm}$ in diameter. The COR with and without confinement are $r_{c}$ and $r$, respectively.

\begin{tabular}{lccc}
\hline \hline Material & $m(\mathrm{~g})$ & $r_{c}$ & $r$ \\
\hline Steel & $2.053 \pm 0.010$ & $0.770 \pm 0.011$ & $0.923 \pm 0.006$ \\
Bronze & $2.235 \pm 0.033$ & $0.746 \pm 0.012$ & $0.861 \pm 0.028$ \\
Brass & $2.383 \pm 0.005$ & $0.742 \pm 0.036$ & $0.860 \pm 0.015$ \\
Nylon & $0.295 \pm 0.006$ & $0.757 \pm 0.011$ & $0.740 \pm 0.042$ \\
Polyurethane & $0.308 \pm 0.008$ & $0.634 \pm 0.024$ & $0.667 \pm 0.029$ \\
\hline \hline
\end{tabular}

is fixed at $200 \mathrm{~Hz}$. Thus, $f_{l}$ and $\Gamma$ serve as control parameters. At the bottom end of the tube a piezoelectric impact sensor (PCB-200B02) is attached to the oscillating piston, making impact with the bead. The impact signal is conditioned via a signal conditioner (PCB-480C02) for data treatment. From the acceleration signal, the velocity of the piston is computed by analog integration of the signal through the signal conditioner. Both force and acceleration signals are digitally acquired at $750 \mathrm{kHz}$, numerically low-passed filtered at $50 \mathrm{kHz}$ to avoid the electrical resonance of the force sensor at $70 \mathrm{kHz}$, and stored in the desktop computer.

\section{A. Coefficient of restitution $r$}

In order to understand the dissipative motion of an inelastic bead bouncing on a randomly moving piston, we first measure the experimental coefficient of restitution (COR) $r$ for different beads of different materials following a scheme similar to the one proposed in Ref. [24]. To do this, the impact sensor is screwed on an aluminum block $\left(10 \times 20 \times 4 \mathrm{~cm}^{3}\right)$, on top of which an aluminum tube (outer diameter $20 \mathrm{~mm}$, inner diameter $15 \mathrm{~mm}$ ) is placed. A micrometric screw holds the bead inside the tube at a height $h_{o}=5 \mathrm{~mm}$ over the impact sensor, allowing a controlled release of the bead with almost negligible air entrainment. The initial impact velocity $v_{o}$ coming from the free falling of the bead can be estimated $v_{o}=\sqrt{2 h_{o} g} \simeq 30 \mathrm{~cm} / \mathrm{s}$. Horizontal and rotational velocities are also negligible for this experimental configuration.

$r$ is defined as the ratio between the bead velocity before and after an impact and it is assumed to be constant [24]. Thus, the COR can be computed as $r=v_{n+1} / v_{n}$. The measurement of $v_{n}$ and $v_{n+1}$ is difficult, but we can compute its ratio using simple particle mechanics relating $r$ with the time of flight of the particle.

\section{B. Calculation of $\boldsymbol{r}$ via time of flight}

The total time elapsed $d_{n}$ between the $n$th and $n+1$ th impact can be measured from the series of force peaks (as the one shown in Fig. 2), which can be used to compute $r . d_{n}$ can be expressed as $d_{n}=\tau_{n}+t_{n}$, where $\tau_{n}$ is the duration of the $n$th collision and $t_{n}$ is the time of flight in between shocks. These times are measured from the temporal trace of the impact sensor, which consists of a series of peaks showing the time events where the bead impacts the sensor bouncing repeatedly over the piston in shortening time intervals as shown in Fig. 3.

Assuming negligible dissipation in between these intervals the ball is performing parabolic flights lasting $t_{n} . d_{n}$ is computed as the temporal difference between two consecutive maxima of the force signal (discriminating between the real maxima and those arising from the piezoelectric inertial oscillation with a temporal period $\sim 100 \mu \mathrm{s}) . \tau_{n}$ is computed as the temporal width of the impact curve (which is always smaller than $\sim 100 \mu \mathrm{s}$ ). Thus, $\tau_{n} \ll t_{n}$ and $d_{n} \simeq t_{n}$. We compute the COR from the set of $t_{n}$ using point particle dynamics [24]:

$$
t_{n}=r \times t_{n-1}=r^{n} \times \sqrt{\frac{8 h_{o}}{g}} .
$$



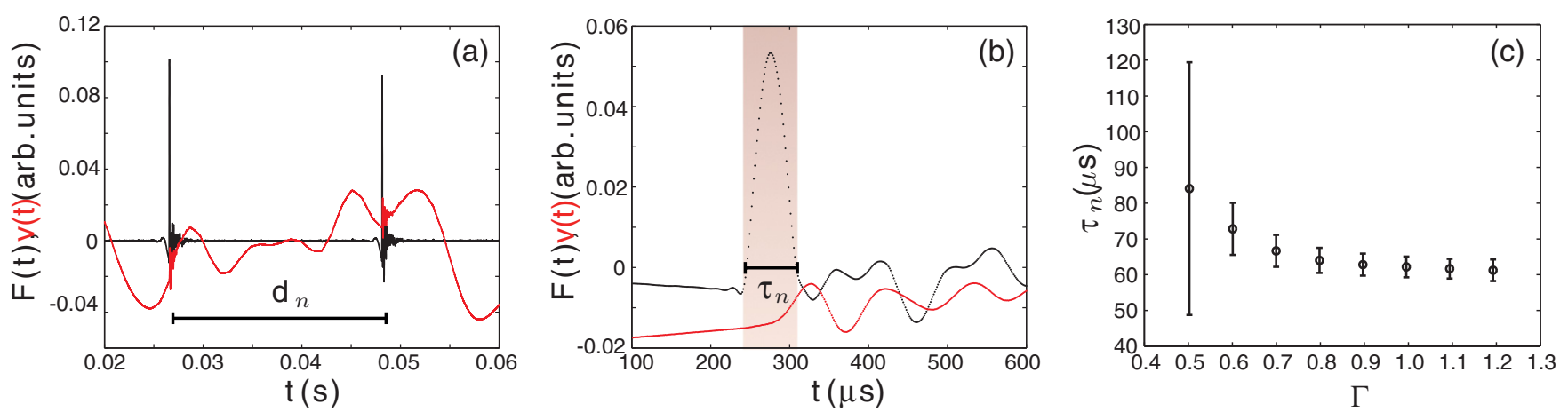

FIG. 2. (Color online) (a) Temporal traces for the force (black) and velocity (red) traces between two impacts, defining $d_{n}$ for $\Gamma=1.09$. (b) Temporal traces for the force (black) and velocity (red) traces, around an impact for $\Gamma=1.1$. The shaded region defines $\tau_{n}$. Arbitrary units are used to place both curves in the same scale. (c) Dependence of $\tau_{n}$ as a function of $\Gamma$. Error bars stand for the standard deviation for $\tau_{n}$. The bead is made of steel.

\section{Dissipation effects}

As stated above, in this approximation, no viscous drag, sliding friction, or velocity-dependent effects on $r$ are taken into account. Taking the natural logarithm on both sides we compute $r$ as the slope of $\log t_{n}$ versus $n$, as is shown in Fig. 3. $r$ is defined as the mean slope of five different trials. This procedure was used to compute the COR for five different materials, as presented in Table I. The COR was also measured following the same scheme but using the enclosing tube to constrain the bead horizontal dynamics. This shifts $r$ values to lower ones, as air entrainment becomes important and even dominant in the bead dynamics. Values for the COR with $\left(r_{c}\right)$ and without confinement $(r)$ are shown also in Table I.

The difference between $r$ and $r_{c}$ appears as a combination of the effect of air entrainment and wall friction due to confinement, affecting the measurement of the COR as the initial and terminal velocity after a bounce of the inelastic ball. In the literature, the problem of the terminal speed change due to confinement (which is responsible for the shift to lower values of $r$ ) has been extensively studied for different tube and

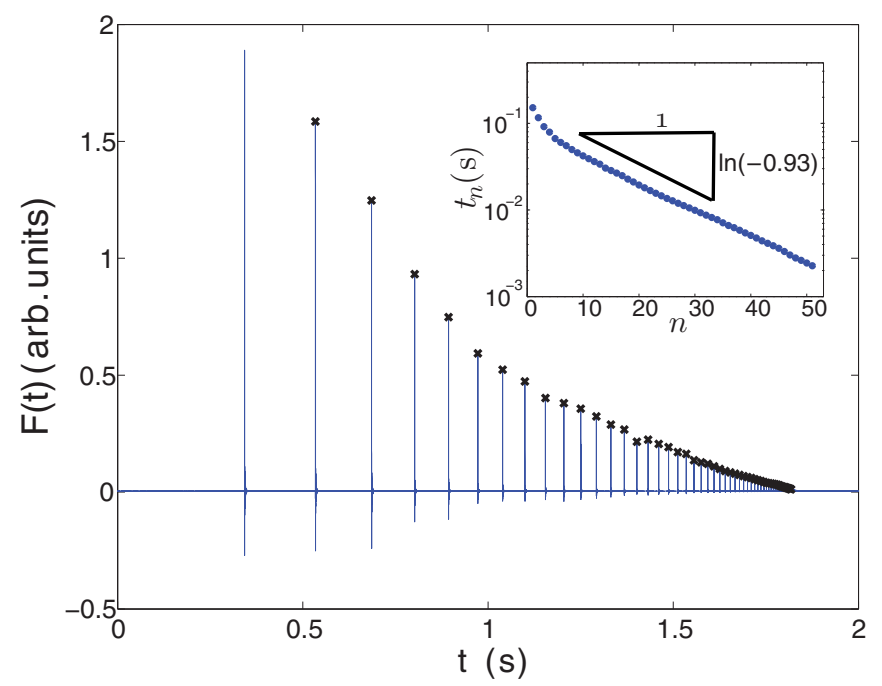

FIG. 3. (Color online) Typical trace of force impacts of a steel ball on a piston on repose. Symbols $(x)$ stand for peak detections. Inset: Semilog plot of time lag between impacts $t_{n}$ vs $n$ for a steel ball. Best fitting slope is $\ln (-0.93)$. particle diameters, terminal speeds, confinement fluids, and flow regimes (see Ref. [25] and references therein). Thus, we will work using the effective $r_{c}$ in what follows and leave this effect for future studies.

In Fig. 2 we show how $d_{n}$ and $\tau_{n}$ are defined from the acquired temporal traces. The values of $d_{n}$ change as piston moves, but its typical scale is still much larger than $\tau_{n}$. The values of $\tau_{n}$ change slightly depending on $\Gamma: \tau_{n}$ is of the order of $50 \mu \mathrm{s}$ when the piston is in repose and $65 \mu \mathrm{s}$ when the piston is moving randomly. These differences are taken into account when computing the discrete values of the exerted force and velocity of the piston, as is shown below.

\section{EXPERIMENTAL RESULTS}

As shown in Fig. 2, a typical trace of the force and velocity for a steel bead $\left(r_{c}=0.770, \Gamma=1.09, f_{l}=0\right)$ displays peaks and oscillations. The force signal consists of a series of peaks separated by the randomly spaced time intervals $t_{n}$ (as explained above). Between these intervals, the ball performs parabolic flights inside the tube. On the other hand, the velocity signal of the piston randomly fluctuates around zero. Around each impact $n$, we compute the discrete force $F_{n}$ and velocity $v_{n}$ by integrating the force and velocity signals in the time interval $\tau_{n}$. Each experimental run lasts $100 \mathrm{~s}$. Between 10 and 500 collisions are observed, depending on the control parameters. To obtain sufficient data points for statistical analysis of all values of the control parameters $\left(\sim 5 \times 10^{4}\right)$, 100 runs are done for each value of these parameters.

\section{A. Statistics of $F_{n}$ and $v_{n}$ as a function of $\Gamma$}

Let us begin by studying the statistics of $F_{n}$ as a function of $\Gamma$ shown in Fig. 4. There is a transition from an exponential to a convexly shaped PDF with increasing $\Gamma$. All the moments of $F_{n}$ increase with increasing $\Gamma$. For large $\Gamma$ the PDF of $F_{n}$ can be fitted accurately by a Gaussian curve with only positive events almost centered at $F_{n}=0$. This effect can be better observed in the normalized PDFs for the rescaled variable $F_{n} / \sigma\left(F_{n}\right)$, displayed in the inset of Fig. 4 (top), where a Gaussian fit is plotted (although the fit is not a normalized Gaussian with standard deviation equal to unity). The bottom of Fig. 4 shows the statistics for $v_{n}$ as a function of $\Gamma$. All the curves can be accurately fitted by a Gaussian curve centered at $v_{n}=0$ 

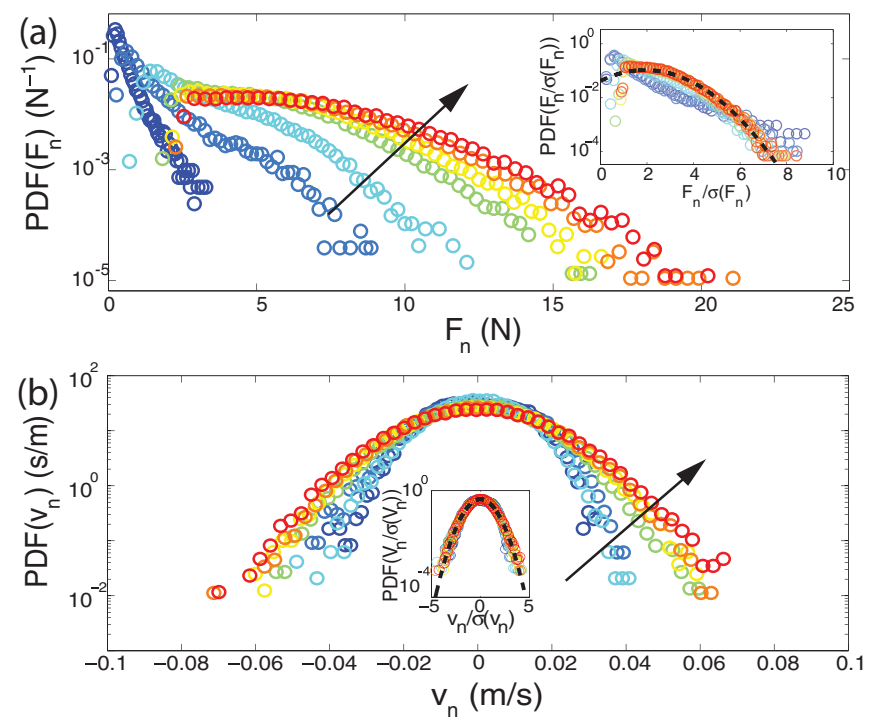

FIG. 4. (Color online) (a) PDF for $F_{n}$ for $\Gamma=0.50,0.60,0.68$, $0.80,0.87,1.0,1.09$, and 1.28 for steel $\left(r_{c}=0.770\right)$ and $f_{l}=0 \mathrm{~Hz}$. Inset: PDF of the normalized variable $F_{n} / \sigma\left(F_{n}\right)$ for $\Gamma=0.50,0.60$, $0.68,0.80,0.87,1.00$, and 1.09 for steel $\left(r_{c}=0.770\right)$ and $f_{l}=0 \mathrm{~Hz}$. (b) PDF of $v_{n}$ for $\Gamma=0.50,0.60,0.68,0.80,0.87,1.0$, and 1.09 for steel $\left(r_{c}=0.770\right)$ and $f_{l}=0 \mathrm{~Hz}$. Inset: PDF of the normalized variable $v_{n} / \sigma\left(v_{n}\right)$ for $\Gamma=0.50,0.60,0.68,0.80,0.87,1.0,1.09$, and 1.28 for steel $\left(r_{c}=0.770\right)$ and $f_{l}=0 \mathrm{~Hz}$. Dashed line is a normalized Gaussian. Arrows indicate how $\Gamma$ increases.

with a standard deviation growing linearly with $\Gamma$. As for $F_{n}$, this effect can be better observed in the normalized PDFs for the rescaled variable $v_{n} / \sigma\left(v_{n}\right)$ shown in the inset of Fig. 4 (bottom), where a normalized Gaussian curve is plotted to emphasize this dependence.

\section{B. Statistics of $\boldsymbol{F}_{\boldsymbol{n}}$ and $\boldsymbol{v}_{\boldsymbol{n}}$ as a function of $\boldsymbol{r}_{\boldsymbol{c}}$}

Fixing now $\Gamma$ at 1.00 and $f_{l}=0 \mathrm{~Hz}$, we change the bead material. Five different materials were used with different values of $r_{c}$ (see Table I). The PDFs of $F_{n}$ for different values of $r$ are shown in Fig. 5 (top). A large difference between metallic and plastic beads is found in the typical values of $F_{n}$. The rms value of metallic beads is 10 times larger than the one for plastic ones. In the normalized variable $F_{n} / \sigma\left(F_{n}\right)$, the PDFs do not collapse on the same curve [see inset of Fig. 5 (top)]. The order of the curves is nonmonotonic with $r_{c}$. The PDFs of $v_{n}$ for different values of $r_{c}$ are shown in Fig. 5 (bottom). The different curves are very similar in scale and shape. A slight asymmetry towards positive values of $v_{n}$ can be observed on the curves when plotted in the rescaled variable $v_{n} / \sigma\left(v_{n}\right)$ [see Fig. 5 (bottom)], as can be seen when compared to a normalized Gaussian curve.

\section{Statistics of $F_{n}$ and $v_{n}$ as a function of $f_{l}$}

Fixing now $\Gamma$ at 1.00 and using a brass bead $\left(r_{c}=0.742\right)$, we change the low-frequency cutoff $f_{l}$ from 0 to $150 \mathrm{~Hz}$. The PDFs of $F_{n}$ for different values of $f_{l}$ are shown in Fig. 6 (top). As $f_{l}$ increases, the typical rms fluctuations of $F_{n}$ decrease and new local maxima appear in the PDFs. These maxima are related to the appearance of a pseudoperiodic motion of the


FIG. 5. (Color online) (a) PDF for $F_{n}$ for steel (o), brass $(\times)$, bronze $(\square)$, nylon $(\diamond)$, and polyurethane $(+)$ for $\Gamma=1.00$ and $f_{l}=0$ Hz. Inset: PDF for the normalized variable $F_{n} / \sigma\left(F_{n}\right)$ for steel (०), brass $(\times)$, bronze $(\square)$, nylon $(\diamond)$, and polyurethane $(+)$ for $\Gamma=1.00$ and $f_{l}=0 \mathrm{~Hz}$. (b) PDF for $v_{n}$ for steel (०), brass $(\times)$, bronze $(\square)$, nylon $(\diamond)$, and polyurethane $(+)$ for $\Gamma=1.00$ and $f_{l}=0 \mathrm{~Hz}$. Inset: PDF for the normalized variable $v_{n} / \sigma\left(v_{n}\right)$ for steel $(\circ)$, brass $(\times)$, bronze $(\square)$, nylon $(\diamond)$, and polyurethane $(+)$ for $\Gamma=1.00$ and $f_{l}=$ $0 \mathrm{~Hz}$.

bead as the bandwidth of the forcing becomes narrower around $200 \mathrm{~Hz}$ (the upper cutoff frequency of the selected bandwidth). When plotted in the rescaled variable $F_{n} / \sigma\left(F_{n}\right)$, the curves
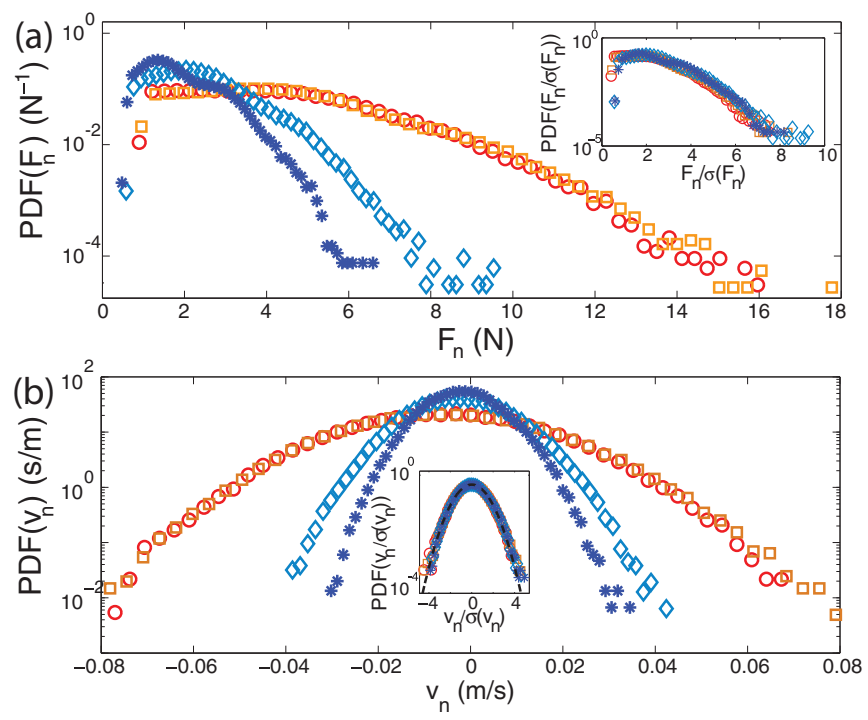

FIG. 6. (Color online) (a) PDF for $F_{n}$ for $f_{l}=0$ (०), 50 ( $\square$ ), 100 $(\diamond)$, and $150(*) \mathrm{Hz}$ for $\Gamma=1.00$, and $r_{c}=0.742$ (brass). Inset: PDF for the normalized variable $F_{n} / \sigma\left(F_{n}\right)$ for $f_{l}=0(\circ), 50(\square), 100(\diamond)$, and $150(*) \mathrm{Hz}$ for $\Gamma=1.00$ and $r_{c}=0.742$ (brass). (b) PDF for $v_{n}$ for $f_{l}=0(\circ), 50(\square), 100(\diamond)$, and $150(*) \mathrm{Hz}$ for $\Gamma=1.00$ and $r_{c}=0.742$ (brass). Inset: PDF for the normalized variable $v_{n} / \sigma\left(v_{n}\right)$ for $f_{l}=0(\circ), 50(\square), 100(\diamond)$, and $150(*) \mathrm{Hz}$ for $\Gamma=1.00$ and $r_{c}=0.742$ (brass). 
present a great level of collapse, although not complete. The PDFs of $v_{n}$ for different values of $f_{l}$ are shown in Fig. 6 (bottom). They are similar in shape, and their respective rms fluctuations decrease with increasing $f_{l}$. A slight asymmetry can be observed on the curves when plotted in the rescaled variable $v_{n} / \sigma\left(v_{n}\right)$ [see Fig. 6 (bottom)], as can be seen when compared to a normalized Gaussian curve.

\section{Injected power $I_{n}$ statistics as a function of $\Gamma, r$, and $f_{l}$}

Using the discrete force $F_{n}$ exerted by the piston on the bead and velocity $v_{n}$ of the piston, we can compute the discrete power $I_{n}=F_{n} \times v_{n}$ injected on the bouncing bead system by the piston as a function of the nondimensional rms acceleration $\Gamma$, the COR $r_{c}$ and the lower cutoff frequency $f_{l}$, as is shown in Fig. 7. Following the scheme presented above, we first fixed $r_{c}=0.770$ and $f_{l}=200 \mathrm{~Hz}$, and we computed
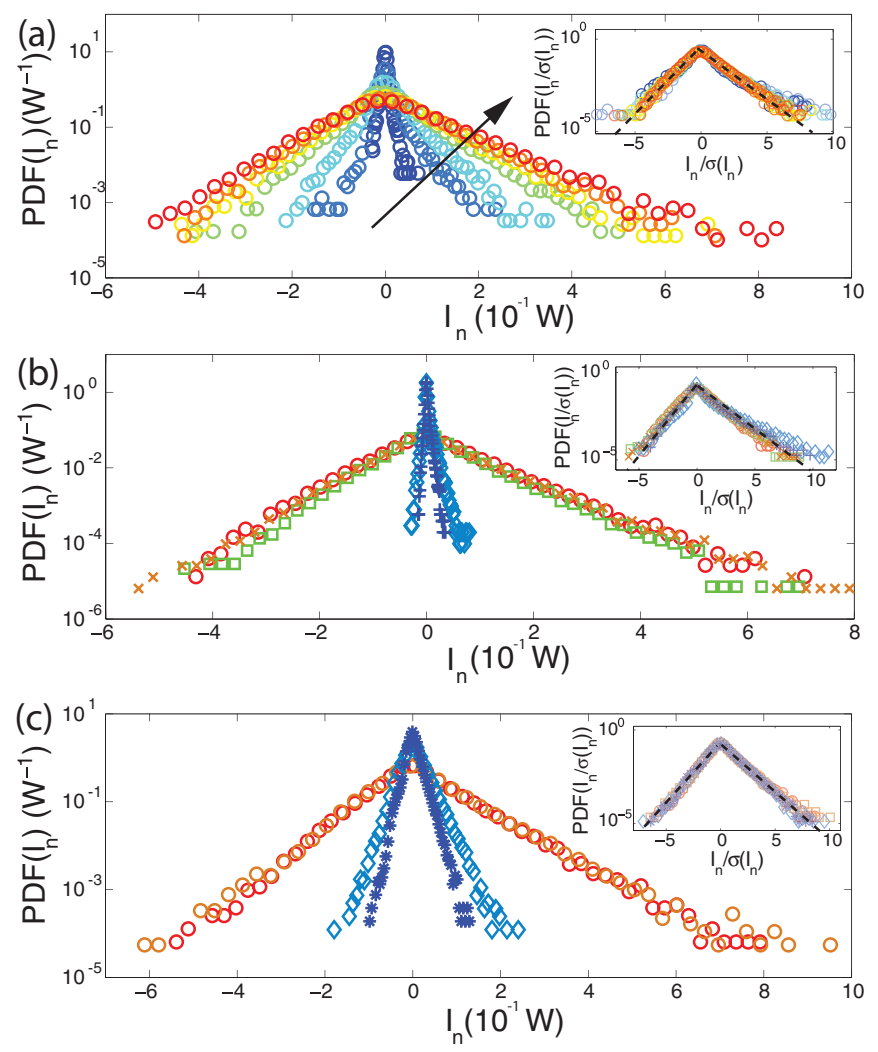

FIG. 7. (Color online) (a) PDF for $I_{n}$ for $\Gamma=0.50,0.60,0.68$, $0.80,0.87,1.00$, and 1.09 for steel $\left(r_{c}=0.770\right)$ and $f_{l}=0 \mathrm{~Hz}$. Inset: PDF of the normalized variable $I_{n} / \sigma\left(I_{n}\right)$ for $\Gamma=0.50,0.60$, $0.68,0.80,0.87,1.00$, and 1.09 for steel $\left(r_{c}=0.770\right)$ and $f_{l}=0$ Hz. Dashed lines are exponential fits for both positive and negative tails. (b) PDF for $I_{n}$ for steel (०), brass $(\times)$, bronze $(\square)$, nylon $(\diamond)$, and polyurethane $(+)$ for $\Gamma=1.00$ and $f_{l}=0 \mathrm{~Hz}$. Inset: PDF for the normalized variable $I_{n} / \sigma\left(I_{n}\right)$ for steel (०), brass $(\times)$, bronze $(\square)$, nylon $(\diamond)$, and polyurethane $(+)$ for $\Gamma=1.00$ and $f_{l}=0 \mathrm{~Hz}$. Dashed lines are exponential fits for both positive and negative tails. (c) PDF for $I_{n}$ for $f_{l}=0(\circ), 50(\square), 100(\diamond)$, and $150(*) \mathrm{Hz}$ for $\Gamma=1.00$ and $r_{c}=0.742$ (brass). Inset: PDF for the normalized variable $I_{n} / \sigma\left(I_{n}\right)$ for $f_{l}=0(\circ), 50(\square), 100(\diamond)$, and $150(*) \mathrm{Hz}$ for $\Gamma=1.00$ and $r_{c}=0.742$ (brass). Dashed lines are exponential fits for both positive and negative tails. the statistics of $I_{n}$ by changing $\Gamma$. The PDFs display a cusp center at $I_{n}=0$ and tails which are asymmetrical; i.e., the probability of finding positive injected power events $\left(I_{n}>0\right)$ is larger than the probability of finding negative injected power events $\left(I_{n}<0\right)$. This means that the mean injected power $\left\langle I_{n}\right\rangle$ is positive for every experimental run, which is needed in out-of-equilibrium systems as power will later be dissipated by internal mechanisms. For very low values of $\Gamma$, the tails decrease slower than an exponential (the convexity of the slopes is positive). As $\Gamma$ increases the slopes become exponential and the typical scale of the fluctuations of $I_{n}$ increases. In the rescaled variable $I_{n} / \sigma\left(I_{n}\right)$ the curves collapse into a master curve which displays exponential tails. When we fix $\Gamma=1.00$ and $f_{l}=200 \mathrm{~Hz}$ and we change $r_{c}$, the PDFs of $I_{n}$ display a large difference between metallic and plastic beads (as in the case for $F_{n}$ ). The rms value of $I_{n}$ for metallic beads is 10 times larger than the one for plastic ones. In the normalized variable $I_{n} / \sigma\left(I_{n}\right)$, the PDFs collapse on the same curve [see inset of Fig. 7 (top)], displaying asymmetric exponential tails and a cusp close to $I_{n}=0$. Fixing $\Gamma$ at 1.00 and $r_{c}$ at 0.742 , we change $f_{l}$ from 0 to $150 \mathrm{~Hz}$. As before, the PDFs of $I_{n}$ for different values of $r_{c}$ are similar in shape, and their respective rms fluctuations decrease with increasing $f_{l}$. In the rescaled variable $I_{n} / \sigma\left(I_{n}\right)$ all curves collapse into the same master curve, which displays exponential asymmetric tails.

In computing $I_{n}$ we have used the discrete multiplication instead of the integral over the collision time $\tau_{n}$ of the product of force and velocity. The statistics of either computation method are almost equal, except for $\Gamma<0.6$ where the cusp near $I_{n}$ is rounder and smaller. This means that the approximation of $I_{n}$ as the direct multiplication of $F_{n}$ and $v_{n}$ is a valid choice. In this framework, the features of this PDF can be understood, as is shown in the next section.

\section{FEATURES OF THE INJECTED POWER STATISTICS}

A way to understand the qualitative features of the PDF of injected power is to adapt the simple model proposed in Ref. [13] following Craig's XY distribution [26]. From the acquired data, we can see that the fluctuations of $v_{n}$ can be described accurately by a Gaussian random variable with zero mean. The fluctuations of $F_{n}$ cannot be described as the fluctuations of $v_{n}$, as no negative values of $F_{n}$ can be achieved, even though the positive $F_{n}$ events are well fitted by a Gaussian curve. Thus, from the data presented above, $F_{n}$ can be approximated a semi-Gaussian variable, i.e., a random variable whose PDF is the product of a Gaussian and a Heaviside function (suitably normalized). We can model the PDF of $F_{n}$, $P\left(F_{n}\right)$ as

$$
P\left(F_{n}\right)= \begin{cases}\sqrt{\frac{2}{\pi}} \frac{1}{\alpha} \exp \left(-F_{n}^{2} / 2 \alpha^{2}\right), & \text { for } F_{n} \geqslant 0 . \\ 0, & \text { otherwise. }\end{cases}
$$

where $\alpha$ is the standard deviation of the Gaussian function if no restriction for negative values of $F_{n}$ is in place. Using the approximation that the exerted force per impact $F_{n}$ drives the inelastic bead into motion, while it changes the momentum of the piston, we calculate the injected power $I_{n}=F_{n} \times v_{n}$ (even in this case where $F_{n}$ is always positive). Here the injected power into the bouncing ball system is computed by 
the multiplication of the velocity and force of the piston, as in Ref. [13]. The PDF of $I_{n}$ can be derived from direct integration of the joint probability distribution function for $F_{n}$ and $v_{n}$ with suitable integration domains.

Let us use a simple model to compute the PDF of $I_{n}$. In the case of two Gaussian variables which are correlated (linear statistical dependence), the joint probability distribution function is a bivariate distribution

$$
P\left(F_{n}, v_{n}\right)=\frac{\exp \left\{-\frac{1}{2\left(1-c^{2}\right)}\left[\frac{v_{n}^{2}}{\sigma\left(v_{n}\right)^{2}}-\frac{2 c v_{n} F_{n}}{\sigma\left(F_{n}\right) \sigma\left(v_{n}\right)}+\frac{F_{n}^{2}}{\sigma\left(F_{n}\right)^{2}}\right]\right\}}{2 \pi \sigma\left(v_{n}\right) \sigma\left(F_{n}\right) \sqrt{1-c^{2}}}
$$

where $c=\frac{\left\langle\left(F_{n}-\left\langle F_{n}\right\rangle\right)\left(v_{n}-\left\langle v_{n}\right\rangle\right)\right\rangle}{\sigma\left(F_{n}\right) \sigma\left(v_{n}\right)}$ is the correlation coefficient for the pair of random variables $\left(F_{n}, v_{n}\right)$. Although other joint distribution functions can be displayed for two Gaussian variables with a given correlation [27], we model our system with this joint distribution because of its simplicity in the calculation of the PDF of $I_{n}$. Changing variables to $I_{n}=$ $F_{n} \times v_{n}$ and $u_{n}=F_{n}$ and integrating Eq. (3) over $F_{n}$ on the domain $[-\infty, \infty]$ we obtain the PDF for $I_{n}$ :

$$
P\left(I_{n}\right)=\frac{\exp \left[\frac{c I_{n}}{\left(1-c^{2}\right) \sigma\left(F_{n}\right) \sigma\left(v_{n}\right)}\right]}{\pi \sigma\left(F_{n}\right) \sigma\left(v_{n}\right) \sqrt{1-c^{2}}} K_{o}\left[\frac{\left|I_{n}\right|}{\left(1-c^{2}\right) \sigma\left(F_{n}\right) \sigma\left(v_{n}\right)}\right],
$$

where $K_{o}(\cdot)$ is the zeroth order modified Bessel function of the second kind. A simple expression of the above equation can be achieved by the steepest descent method,

$$
P(\hat{X}) \sim \exp (c \hat{X}-|\hat{X}|) / \sqrt{|\hat{X}|},
$$

with $\hat{X}=I_{n} /\left[\left(1-c^{2}\right) \sigma\left(F_{n}\right) \sigma\left(v_{n}\right)\right]$. In addition $\left\langle I_{n}\right\rangle=\left\langle F_{n} \times\right.$ $\left.v_{n}\right\rangle=c \times \sigma\left(F_{n}\right) \sigma\left(v_{n}\right)$, which connects the correlation coefficient of both variables, their rms fluctuations, and the injected power into the system. This expression displays two exponential tails controlled solely by the correlation coefficient $c$, which is equal to the mean injected power normalized when $\left\langle v_{n}\right\rangle=0$.

We can obtain an expression for the PDF of $I_{n}$ by assuming that $F_{n}$ is semi-Gaussian and integrating it out in Eq. (3). The new PDF for $I_{n}$ has the same form as Eq. (4) with two modifications: by direct computation $\left\langle F_{n}\right\rangle=\sqrt{2 / \pi} \alpha$ and $\sigma\left(F_{n}\right)=\alpha^{2}(1-2 \pi)$, which are functions of $\alpha$. With these substitutions in Eq. (3), the calculation of $P\left(I_{n}\right)$ is the same as the one above. To wit, we plot in Fig. 8 the PDFs of $I_{n}$ in the reduced variable $X /\left(1-\langle X\rangle^{2}\right)$ with $X=$ $I_{n} \sqrt{1-2 / \pi} /\left[\sigma\left(F_{n}\right) \sigma\left(v_{n}\right)\right]$ and the theoretical approximation for its PDF following Eq. (5). There is a good agreement for every COR between experimental and theoretically predicted curves. As predicted, the approximation to the theoretical PDF is good, although the negative tails depart from the predicted slope slightly for polyurethane. Notice that Eq. (4) uses no fitting parameters: all cumulants of $X$ can be computed by knowing $c$.

\section{DISCUSSION AND CONCLUSIONS}

The discrete injected power $I_{n}$ needed to drive an inelastic bead into a NESS is studied. Power is injected via a randomly accelerating piston that drives the motion of the

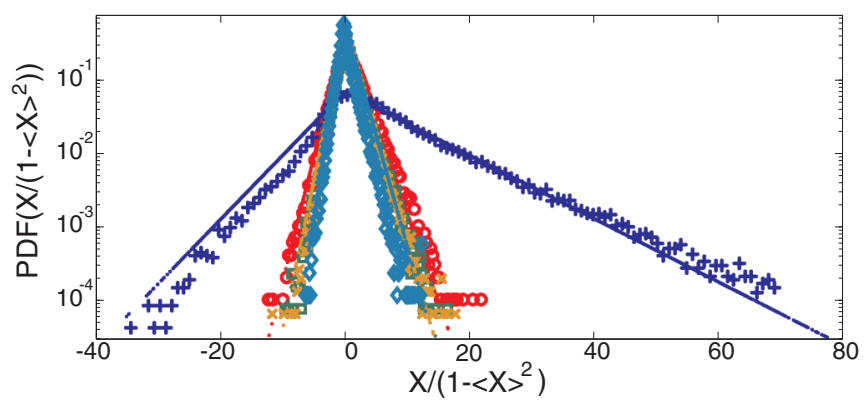

FIG. 8. (Color online) PDF of the reduced variable $X /\left(1-\langle X\rangle^{2}\right)$ with $X=I_{n} \sqrt{1-2 / \pi} /\left[\sigma\left(F_{n}\right) \sigma\left(v_{n}\right)\right]$ for steel (o), brass ( $\times$ ), bronze $(\square)$, nylon $(\diamond)$, and polyurethane $(+)$ for $\Gamma=1.00$ and $f_{l}=0 \mathrm{~Hz}$. Dots show the approximation $P(X) \sim \exp (c X-|X|) / \sqrt{|X|}$ for each value of $r_{c}$.

bead. Measuring both the mean force exerted by the piston over the inelastic bead and the mean velocity of the piston at each impact we can access the complete statistics of $I_{n}$. We showed that by changing the rms fluctuations and frequency band of the random acceleration, and the restitution coefficient of the bead, the typical scale of the fluctuations of $I_{n}$ can change drastically, although the shape of the PDF of $I_{n}$ does not, as it is controlled solely by the normalized injected power $c=$ $\left\langle\left(F_{n}-\left\langle F_{n}\right\rangle\right)\left(v_{n}-\left\langle v_{n}\right\rangle\right)\right\rangle / \sigma\left(F_{n}\right) \sigma\left(v_{n}\right)$. Using a simple model stemming from a bivariate distribution function the PDF can be predicted only knowing $c, \sigma\left(v_{n}\right)$, and $\sigma\left(F_{n}\right)$. This is shown in Fig. 8: as $r_{c}$ is similar for four out of five materials used in the experiment, the PDFs of the normalized injected power display the same shape for a given $\Gamma$ and $f_{l}$ and only for the case of polyurethane $\left(r_{c}=0.634\right)$ shows a large deviation from the other curves. Thus, in this simple out-of-equilibrium case, one can predict and compute the PDF of $I_{n}$ with only a very small set of external parameters which control completely the injected power statistics into an out-of-equilibrium system.

The PDFs exhibited in Fig. 8 are actually quite generic of the injected power furnished by a random force. The simplest system of this type is a single particle submitted to a viscous drag and a random external force. This system can be described as an Ornstein-Uhlenbeck random process (a noise with exponential correlation $[16,17])$. In the case using the joint bivariate normal probability function (3), the PDF of the injected power can be computed exactly [28]. Experimentally, such PDFs have been observed for a randomly vibrated thin elastic plate [12] as well as in randomly forced surface waves [13]. The shape of these PDFs can be also achieved in turbulent shell model submitted to random force [28]. This kind of fluctuations can be observed for other quantities resulting from the product of two centered random variables. The components of the turbulent Reynold stress tensor [29] or the rate of transferred energy in turbulence [30] displays the same features. They also appear in turbulent convection for the local heat transfer, which is mainly the product of temperature and vertical velocity fluctuations. Asymmetric PDFs with a cusp near zero and exponential tails were observed for the temporal fluctuations of the locally measured heat flux [31], for the instantaneous fluctuations in space of the numerically computed heat flux [32], and for the measurement of the heat flux using a Lagrangian probe [33]. There may exist 
other situations where such fluctuations are displayed. Even if the two variables involve in the product are not necessary Gaussian, the expression for the PDF of the injected power (4) is a useful benchmark to study the fluctuations of such a product. Thus, this simple yet powerful approach to the global fluctuations of an out-of-equilibrium system used in other NESS [8,11-13] serves as a tool to study the statistical properties of different systems.

In that sense, the use of this approach can be thought as an opportunity to rethink the pertinence of some of the tools of out-of-equilibrium fluctuations, for instance, fluctuation theorems [34], which are used to compute parameters associated to dissipative constants and effective energy scales (as in the case of the granular temperature $[5,6]$ ). Our work shows that from the statistics of $I_{n}$ we can distinguish what would be an effective dissipation coefficient in a dissipative out-of-equilibrium system. In our experiments, the different masses of the beads do not affect the PDF of the reduced variable $X$, even when the difference is one order of magnitude in mass. Only the different values of $r_{c}$ (which in our case depends on geometrical factors, viscous drag, friction, and plasticity of the material) affect the measured statistics of $X$ as is shown in Fig. 8. Thus, $r_{c}$ is a relevant control parameter of the dissipative system as it controls the amount of power that can be injected into the bouncing bead. The idea of the construction of an effective dissipation coefficient in dissipative out-of-equilibrium systems (such as the case of $r_{c}$ ) can thus be exported to systems where internal dissipative mechanisms are not yet measured or not well understood. Simple models for the statistics of the injected power, as the one presented here or elsewhere [28], can be used to quantify, for instance, typical dissipative scale differences between systems as internal parameters are changed, even though no complete information of the internal microscopic dynamics is known. The application of this scheme on other dissipative out-of-equilibrium systems is then needed to confirm its potential use.

\section{ACKNOWLEDGMENTS}

C.F. thanks Univ Paris Diderot for their Invited Researcher funding in Paris. E.F. thanks Ecos-Chili ECOS C07E07 for their Invited Researcher funding in Santiago. This work has been supported by FONDECYT 1130354, CONICYT FONDECYT Postdoctorado 3140550, ACT127 and European Space Agency Topical Team on granular materials No. 4000103461.
[1] A. N. Kolmogorov, Dokl. Akad. Nauk SSSR 32, 19 (1941); reprinted in Proc. R. Soc. Lond. A 434, 15 (1991).

[2] U. Frisch, Turbulence: The Legacy of A. N. Kolmogorov (Cambridge University Press, New York, 1995).

[3] A. Mehta, Granular Physics (Cambridge University Press, New York, 2007).

[4] I. Aranson and L. Tsimring, Granular Patterns (Oxford University Press, New York, 2009).

[5] S. Aumaître, S. Fauve, S. McNamara, and P. Poggi, Euro. Phys. J. B 19, 449 (2001).

[6] S. Aumaître, J. Farago, S. Fauve, and S. Mc Namara, Euro. Phys. J. B 42, 255 (2004).

[7] N. Garnier and S. Ciliberto, Phys. Rev. E 71, 060101 (2005).

[8] C. Falcón and E. Falcon, Phys. Rev. E 79, 041110 (2009).

[9] M. Bonaldi et al., Phys. Rev. Lett. 103, 010601 (2009).

[10] G. Alhers, S. Grossman, and D. Lohse, Rev. Mod. Phys. 81, 503 (2009).

[11] M. M. Bandi and C. Connaughton, Phys. Rev. E 77, 036318 (2008).

[12] O. Cadot, C. Touzé, and A. Boudaoud, Phys. Rev. E 82, 046211 (2010).

[13] E. Falcon, S. Aumaître, C. Falcón, C. Laroche, and S. Fauve, Phys. Rev. Lett. 100, 064503 (2008).

[14] U. Seifert, Rep. Prog. Phys. 75, 126001 (2012).

[15] S. Ciliberto, S. Douady, and S. Fauve, Europhys. Lett. 15, 23 (1991).

[16] R. Zwanzig, Nonequilibrium Statistical Mechanics (Oxford University Press, New York, 2001).

[17] R. Kubo, M. Toda, and N. Hashitsume, Statistical Physics II: Nonequilibrium Statistical Mechanics, Springer Series in SolidState Sciences (Springer, New York, 1985).
[18] P. Pieranski, J. Physique 44, 573 (1983).

[19] J. M. Luck and A. Mehta, Phys. Rev. E 48, 3988 (1993).

[20] J.-C. Geminard and C. Laroche, Phys. Rev. E 68, 031305 (2003).

[21] S. Giusepponi, F. Marchesoni, and M. Borromeo, Physica A 351, 142 (2005).

[22] C. R. de Oliveira and P. S. Goncalves, Phys. Rev. E 56, 4868 (1997).

[23] T. W. Burkhardt and S. N. Kotsev, Phys. Rev. E 73, 046121 (2006).

[24] E. Falcon, C. Laroche, S. Fauve, and C. Coste, Eur. Phys. J. B 5, 111 (1998).

[25] R. P. Chhabra, S. Agarwal, and K. Chaudhary, Powder Technol. 129, 53 (2003).

[26] C. Craig, Ann. Math. Stat. 7, 1 (1936).

[27] R. Hogg, E. Tanis, and D. Zimmerman, Probability and Statistical Inference (Pearson, New York, 2001).

[28] S. Aumaître, Fluctuations and Instabilities in Out-ofEquilibrium Systems (Habilitation à diriger les recherches (HDR), École Normale Supérieure de Lyon, 2014).

[29] A. Pumir, Phys. Fluids 8, 3112 (1996).

[30] S. Cerutti and C. Meneveau, Phys. Fluids 10, 928 (1998).

[31] X.-D. Shang, X.-L. Qiu, P. Tong, and K.-Q. Xia, Phys. Rev. Lett. 90, 074501 (2003).

[32] O. Shiskina and C. Wagner, Phys. Fluids 90, 085107 (2007).

[33] W. L. Shew, Y. Gasteuil, M. Gibert, P. Metz, and J. F. Pinton, Rev. Sci. Inst. 78, 065105 (2007).

[34] D. J. Evans, E. G. D. Cohen, and G. P. Morriss, Phys. Rev. Lett. 71, 2401 (1993). 